\title{
Black Holes, Quasars, Blazars, and all that... How to explain them to a lay audience?
}

\author{
Hamid Hamidani and Jamal Mimouni \\ Ecole doctorale d'astrophysique, Departement de Physique, \\ Mentouri University \\ Route de Ain Bey, Constantine, 25000 Algeria \\ email: hamidanihamid@yahoo.fr, jamalmimouni@yahoo.com
}

\begin{abstract}
The popularisation of science is central to the culture of the citizen, and if astronomy make poets dream (and many others), astrophysics attracts young and old talents to science. To reveal in simple terms the stellar bestiary from the black holes to the quasars proceeds along this line. Black hole is still the object which is subject to most questions in any public talk on astronomy. How indeed does contemporary physics link the black hole, this stellar gravitational tomb, with the quasar, a galaxy gone mad? The tale is worth telling and makes indeed a beautiful story for contemporary young audience. Can it be told to our high school students with their modest scientific background? This is what we intend to do in this paper, spicing the sauce at the end with some new insights on the unified model of AGN.
\end{abstract}

Keywords. Black holes, quasars, outreach

\section{Black holes: the darkest objects in the universe !}

\subsection{Using Newton's theory}

The first scientist who speculated that black holes could exist was John Michell in 1784 . Using Newton's theory of gravity, Michell calculated the critical conditions of an object that not even light could escape from its gravity. The escape velocity $v_{\text {escape }}$ of an object with a mass $M$ and a radius $R$ is simply given by:

$$
v_{\text {escape }}=\sqrt{\frac{2 G M}{R}} \Longrightarrow \quad R=\frac{2 G M}{v_{\text {escape }}^{2}}
$$

where $G$ is the gravitational constant. We then deduce the critical radius $R_{\text {critical }}$ for which the escape velocity becomes equal to the velocity of light: $R_{\text {critical }}=2 \mathrm{GM} / \mathrm{c}^{2}$, $c$ being the speed of light. Since nothing can be faster than light, nothing (matter or radiation) could escape from inside an object with a mass $M$ and radius $R_{\text {critical }}$.

\subsection{Einstein's General Theory of Relativity}

Einstein's General Theory of Relativity (1905) enables us to describe more accurately so black holes which turn into even more fascinating objects. The critical radius of a black hole in General Relativity theory (GR) is called the Schwarzschild radius: $R_{\text {Sch }}=$ $2 G M / c^{2}$. It is only a happy coincidence that the computation of the critical radius of a black hole in GR gives the same result as in Newton's theory!

\subsection{The horizon of the black hole}

The horizon of a black hole is an imaginary sphere of radius $R_{S c h}$ which represents the border between what is still in our universe and the black hole's universe. All what goes inside this sphere will be imprisoned by the black hole's gravity force and will ultimately end up at its centre. 


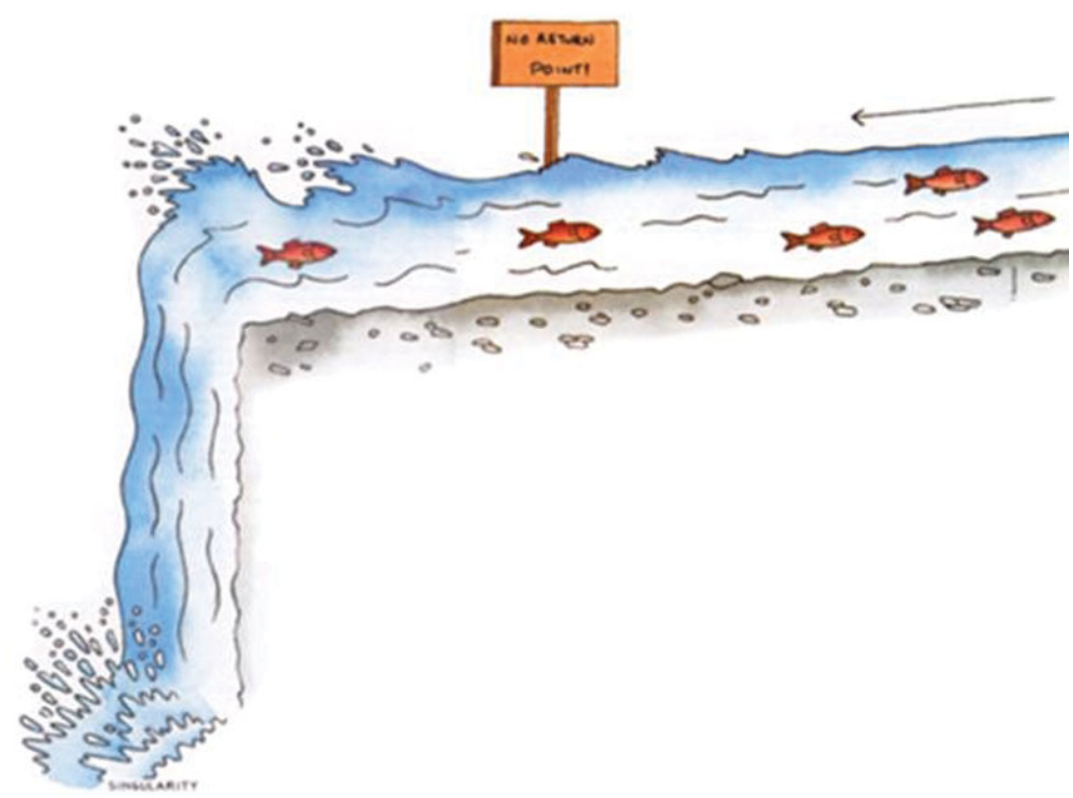

Figure 1. W. Unruh's analogy of the critical radius of a black hole with a water fall.

\subsection{The no return point}

An analogy proposed by William Unruh of the University of British Columbia, illustrates rather well the nature of the horizon. Let's consider the course of a river which ends by a cascade. The fastest fish which lives there is: let say the fish light. Along a line with the approach of the cascade, the current is as fast as the fish is; any fish light which exceeds this line cannot go up the current any more, it is condemned to be crushed against the rocks, in the falls (the singularity in this case); nevertheless the fish-light without will not feel anything when it passes the no return point: neither running nor shock waves will inform it of the passage of this point

\subsection{Formation of a stellar black hole}

A star is the result of a delicate equilibrium between the energy production at its centre which attempt to make the star explode, and the gravitational pressure which tries to compress the star and make it implode. After having exhausted all the nuclear fuel in the central region which provides the internal pressure, nothing will be able to counter this unrelenting gravitational grasp, and an implosion will occur whereby the iron core of the star will cave in a movement close to a freefall, followed by a supernova explosion. If the mass of the initial core of star is higher than some 3.3 solar masses, then it will fall indefinitely towards the centre and the density in the center of star will becomes extreme, so a singularity in space time will be produced which constitutes a stellar black hole.

\subsection{Supermassive black holes at the center of galaxies}

After several years of observation of stars' movements near the centre of our Galaxy, astronomers noticed that these stars revolved with considerable velocities around an empty point in space. The simple application of Kepler's third law to the movement of those stars enable us to deduce the mass of this object which was estimated to be equivalent to some 3 million solar masses. Such an invisible object could only be a massive 
Table 1. Is the universe the ultimate black hole?

\begin{tabular}{cccl}
\hline Object & Critical radius & Density $\left[\mathrm{gcm}^{-3}\right]$ & Note \\
\hline Human $(90 \mathrm{~kg})$ & $1.410^{-25} \mathrm{~m}$ & $\sim 8.10^{72}$ & $10^{58}$ times the nuclear density \\
Sun & $3 \mathrm{~km}$ & $\sim 2.10^{16}$ & 50 times the nuclear density \\
Quasar $\left(310^{9} M_{\odot}\right)$ & 1 light-year & $\sim 2.10^{-9}$ & a billion times less than water \\
Universe & $10^{10}$ light-years & $\sim 10^{-29}$ & observed density \\
\hline
\end{tabular}

black hole. From Kepler's third law:

$$
\frac{T^{2}}{R^{3}}=\frac{4 \pi^{2}}{G M}
$$

We can deduce the mass $M$ of the attracting center -the Black Hole- knowing the radius $R$ of the orbit of the revolving star, and its rotation period $T$. Other astrophysical observations showed that many galaxies have a supermassive black hole at their centres which release a tremendous amount of energy that powers the spectacular energetic events that go on within these galaxies. For example, the black hole at the centre of the M87 galaxy has been calculated to have the mass of some 3 billion Suns !

\subsection{How do massive black holes get bigger?}

The only way for a black hole to get bigger is to swallow up gas and stars, so a supermassive black hole is a fat stellar black hole which by swallowing matter, has grown to millions or even in some cases billions of times the mass of the Sun.

\subsection{Black holes density}

The average density of any black hole is given by: $\rho=M / V=M /(4 / 3) \pi R^{3}$, where $V$ is the volume of the black hole and $R$ is its radius. If $R$ increases, the density decreases, so super massive black holes have a lower average density than a stellar black hole. For example the density of the Milky Way's supermassive black hole is lower than the density of water!

\subsection{Is the universe the ultimate black hole?}

Any object with a mass $M$ has a critical radius, whereby if this object is compressed past its critical radius, the object collapses under the effect of its own gravity till reaching a null volume and an infinite density. It is rather funny that computing the density of the Universe within its critical radius gives a value close to the measured density of the Universe. If the Universe is finite (not yet established), and if its mass is contained within a radius of some ten billion light-years, then we are trapped in the horizon of the Universe-black hole and we cannot go out so that the universe will finish collapsing!

\section{Quasars, Blazars and AGNs, the brightest objects in the universe!}

\subsection{Quasars and blazars: the new beasts}

Quasars are farther away from Earth than any other known object in the Universe, so it takes billions of years for the light to reach Earth. So when we look at quasars, it is like we are looking back in time. But even at such huge distances, quasars are still brilliant, so they should give off huge amounts of energy and can indeed be a trillion times brighter than our Sun! The central engine of quasars which gives them such an enormous energy output is believed to be a super massive black hole at their centres as we argued earlier. Such an object should consume the matter at the rate of some 10 stars per year. It is 
estimated the most brilliant quasars devours some 1000 solar matter masses per year! It is the accretion of matter by the super massive black hole which produces extremely violent jets of energy and particles that we detect on Earth. Blazars are similar to quasars except that their luminosity can vary rapidly, in some case by a factor of 100 in only few days! A blazar is believed to be a quasar which has one of its jets pointed towards Earth so what we observe is primarily emission from the jet region. Any slight variation of the jet's direction will lead to large change of detected energy which explains its extreme variability.

\subsection{The unification model of active galaxies}

Active galaxies are galaxies that have a strong brightness from their nucleus that cannot be explained by the stars' energy production. Models of active galaxies suggest the presence of a super massive black hole at the centre of these galaxies. The galaxy provides the black hole with stars and dust, while the accretion of this matter by the black hole produces a huge flow of energy. The unification model of active galaxies proposes that there is a single type of physical object observed under various conditions. The differences between various types of objects are simply due to different orientations; this model unifies active galactic nuclei with blazars and quasars. Most scientists believe that, even though these types look very different to us, they are all the same thing viewed from different directions. Quasars are active galaxies which are far away from us. Blazars are like quasars but with jets pointed towards the Earth. On the other hand, if the jet is not pointing towards the Earth at all, we will see a galaxy with active nuclei.

\section{Synthesis}

Black holes are possibly the most fascinating objects in the universe. We find them in the Universe with different densities, at different distances and with different sizes, and curiously enough, even the Universe as a whole looks strangely as an extrapolated black hole with a radius equal basically to the Hubble radius, the ultimate black hole as it were. On the other hand, black holes play a fundamental rôle in the evolution of the universe, especially in the evolution of galaxies, by forming their nuclei. On the paradoxical side, black holes are theoretically the darkest objects in the universe, yet thanks to the accretion disk physics powered by their strong gravitational fields, they are the brightest objects in the universe. It is this grand vision that we wished to bring forth in this paper, a grand vision linking the spatiotemporal well that the black hole represents, with the fate of the most primary objects of the universe the stars; the most powerful objects known, the AGNs; the Universe's structuration itself; the formation of its basic units, the galaxies; and perhaps even the description of the Universe as the ultimate Black Hole.

\section{References}

Antonucci, R. 1993, ARAA, 31, 473

Celotti, A., Miller, J. C., \& Sciama, D.W. 1999, Classical and Quantum Gravity, 16, A3

Ghez, A. M. 2005, ApJ, 620, 744

Luminet, J. P. 1992, Les trous noirs (Paris: Seuil)

Thorne, K. S. 1994, Trous noirs et distorsions du temps (Paris: Flammarion) 